\title{
Additional acceleration of solar-wind particles in current sheets of the heliosphere
}

\author{
V. Zharkova ${ }^{1}$ and O. Khabarova ${ }^{2}$ \\ ${ }^{1}$ Department of Mathematics and Information Systems, Northumbria University, Newcastle upon Tyne, NE2 1XE, UK \\ ${ }^{2}$ Heliophysical Laboratory, Institute of Terrestrial Magnetism, Ionosphere and Radiowave Propagation RAS (IZMIRAN), \\ Troitsk, Moscow, 142190 Russia
}

Correspondence to: V. Zharkova (valentina.zharkova@northumbria.ac.uk)

Received: 17 August 2014 - Revised: 12 January 2015 - Accepted: 5 March 2015 - Published: 9 April 2015

\begin{abstract}
Particles of fast solar wind in the vicinity of the heliospheric current sheet (HCS) or in a front of interplanetary coronal mass ejections (ICMEs) often reveal very peculiar energy or velocity profiles, density distributions with double or triple peaks, and well-defined streams of electrons occurring around or far away from these events. In order to interpret the parameters of energetic particles (both ions and electrons) measured by the WIND spacecraft during the HCS crossings, a comparison of the data was carried out with 3-D particle-in-cell (PIC) simulations for the relevant magnetic topology (Zharkova and Khabarova, 2012). The simulations showed that all the observed particle-energy distributions, densities, ion peak velocities, electron pitch angles and directivities can be fitted with the same model if the heliospheric current sheet is in a status of continuous magnetic reconnection. In this paper we present further observations of the solar-wind particles being accelerated to rather higher energies while passing through the HCS and the evidence that this acceleration happens well before the appearance of the corotating interacting region (CIR), which passes through the spacecraft position hours later. We show that the measured particle characteristics (ion velocity, electron pitch angles and the distance at which electrons are turned from the HCS) are in agreement with the simulations of additional particle acceleration in a reconnecting HCS with a strong guiding field as measured by WIND. A few examples are also presented showing additional acceleration of solarwind particles during their passage through current sheets formed in a front of ICMEs. This additional acceleration at the ICME current sheets can explain the anticorrelation of ion and electron fluxes frequently observed around the ICME's leading front. Furthermore, it may provide a plau-
\end{abstract}

sible explanation of the appearance of bidirectional "strahls" (field-aligned most energetic suprathermal electrons) at the leading edge of ICMEs as energetic electrons generated during a magnetic reconnection at the ICME-front current sheet.

Keywords. Ionosphere (particle acceleration)

\section{Introduction}

Magnetic reconnection is actively used to explain diverse solar phenomena, such as flares, coronal mass ejections (CMEs), post-flare or CME loops, coronal jets, blobs, and the restructuring of the solar atmosphere, in general, as well as coronal heating and impulsive solar energetic-particle events. A reconnection plays a central role in the interpretation of a wide variety of observed solar, space, astrophysical and laboratory plasma phenomena (e.g. Priest and Forbes, 2000).

Most researchers agree that the energy in these events comes from the energy of a reconstructed magnetic field, which is then converted into the energy of associated events (shocks, energetic particles, radiation, etc.). However, the exact ways in which this energy is converted into one form or the other are not fully clear yet despite great efforts by many researchers; this is because their diagnostics, say, in the solar corona, are very often obscured by other effects (such as particle, radiation or wave transport through the media) before they are registered by instruments. In this sense, observations of magnetic reconnection from in situ measurements bring much more clarity to understanding this process, provided the measurements are reliable and can be traced again if required. 
Of course, particles in the solar wind can be accelerated by shocks, both quasi-parallel and quasi-perpendicular, that have been the subject of a good amount of research (see, e.g., Reames, 1999, 2009; Neergaard Parker et al., 2014; Neergaard Parker and Zank, 2014, and references therein). We acknowledge the importance of such acceleration as well as the problems which come with it with regard to shocks being formed by certain processes and for particles to access these shocks in order to be consequently accelerated by them. We fully support this mechanism of additional acceleration in solar-wind particles for some observational cases, while in other instances particle energisation can be associated with certain topological changes in the magnetic field which cannot be provided by the shocks. The latter cases encourage researchers to assume that there must be additional acceleration of the solar-wind particles which occurs during a reconnection process in the heliospheric current sheet (Zharkova and Khabarova, 2012) or in other current sheets in the heliosphere.

There are numerous observations of reconnection events in the solar-wind current sheets made by the Advanced Composition Explorer (ACE) and Helios (Gosling et al., 2006; Gosling, 2007), WIND (Phan et al., 2010) and Cluster (Chian and Muñoz, 2011) spacecraft as well as by combined instruments (Gosling, 2009; Gosling and Phan, 2013, and references therein). Many current sheets occur in the heliosphere at the leading edges of interplanetary coronal mass ejections (ICMEs) (see, for example, Xu et al., 2011, and references therein), although, the trigger mechanism initiating these reconnection events is not fully understood.

In situ observations by WIND (Phan et al., 2010) have revealed 34 reconnection sites during $1358 \mathrm{~h}$ of continuous observations of high-speed solar-wind data with the flow exhausts embedded within sharp outward-propagating Alfvénic fluctuations. The authors reported very localised locations of reconnection $X$ lines and recorded the local shear angles across the exhausts as ranging from 24 to $160^{\circ}$ (with the average value about $90^{\circ}$ ). The width of these exhausts was less then $4 \times 10^{4} \mathrm{~km}$, a distance which the satellite can cover in under $100 \mathrm{~s}$.

These current sheets in the solar-wind environment can be associated with streamers and filaments and can be produced by turbulence, created during the solar wind's ejection from the corona, while smaller-scale current sheets observed at several AU may originate from in situ turbulence in the solar wind (Greco et al., 2010; Lazarian et al., 2012). However, some alternative suggestions can be also considered, such as these current sheets being caused by certain processes in the interplanetary space, e.g. by the interaction of the magnetic field in the heliospheric current sheet (HCS) or by the interaction of the interplanetary magnetic field (IMF) with the ICMEs.

Under certain conditions, a magnetic reconnection process can be triggered and maintained for a sufficiently long time. For example, Swisdak et al. (2010) investigated theoretically the conditions of magnetic reconnection in the heliopause and showed that the reconnection can only occur if the interstellar magnetic field points to the heliospheric field in a nearly antiparallel way; this can stabilise the process of reconnection and secure steady streams of ions from the current sheet, which induces large diamagnetic drifts in the reconnecting plasma.

These conditions can be applied to any current sheets formed in the interplanetary space, as has been demonstrated by further observations (Phan et al., 2010). The authors managed to catch the onset of magnetic reconnection in 197 events recorded by the WIND spacecraft and to deduce that the conditions when the reconnection can start and continue to exist are dictated by two factors: the difference in plasma beta on the two sides of the current sheet, which has to be small, and the presence of a magnetic shear across the current sheet as predicted by Swisdak et al. (2010).

The observations with the Cluster satellites of an ICME recorded on the 21 January 2005 as reported by Chian and Muñoz (2011) revealed the two reconnecting current sheets that occurred at the edge of the ICME, which had a multifractal scale of turbulence with a plateau in the magnetic field component in the middle of the current sheet. This plateau is related to the region of the reconnection jets predicted by Swisdak et al. (2010) and propagating flows of Alfvén waves, similar to those reported by McComas et al. (1995) and Gosling et al. (2011).

However, it is not yet known exactly how and where these current sheets are formed in the solar wind. It is expected that passing ICMEs can trigger magnetic reconnections in the HCS or turbulent magnetic fields, which produce these smaller current sheets in the interplanetary space, leading to a stream of energetic particles in the fast solar wind. These conditions still need to be investigated theoretically and observationally for given magnetic topologies and physical conditions relevant to ICMEs, turbulent magnetic fields and the magnetopause.

In addition to direct in situ measurements of magnetic field components in the heliosphere, good candidates that may help to investigate magnetic reconnection processes are the solar-wind particles additionally accelerated while passing through reconnecting current sheets. By exploiting the highenergy particle distributions in density, energy and pitch angles, it is possible to trace the processes of their energisation and movements for a given magnetic field topology in reconnecting current sheets. This approach was shown to work reasonably well in a single heliospheric current sheet, as was proven by Zharkova and Khabarova (2012), allowing the authors to uncover some intriguing dynamics of both ions and electrons, which were fitted fairly closely by the theoretical prediction of the particle-in-cell (PIC) approach.

Furthermore, this approach helped to explain the longstanding controversy in the definition of the locations of sector boundaries in the HCS. Crooker et al. (2004) have noted, from the electron pitch angle spectrograms, that the moments 
when the magnetic field components change their signs, normally assigned to a current sheet origin ("the classic" sector boundary, or the heliospheric current sheet midplane), differ from the times when electrons change their angles by $180^{\circ}$, or make "U turns". In the other words, there are noticeable delays (from 25 min up to $8 \mathrm{~h}$ ) for the electron pitch angle changes in comparison with the change in magnetic field sign; the locations of the $\mathrm{U}$ turns of electrons were designated by Crooker et al. (2004) as the "revised" sector boundaries.

However, the study by Zharkova and Khabarova (2012) showed with 3-D model simulations that the sector boundary, or the HCS origin, should occur, as expected, at the locations where the sign of the magnetic field changes, while the $\mathrm{U}$ turn of electrons at some distance from this location is caused by electron magnetisation in a strong guiding field of the HCS. In the other words, a fraction of electrons (bounced ones) are so greatly magnetised by the strong guiding field that they cannot reach its midplane, where they would gain more energy from a reconnection electric field. Instead, these electrons are turned back by $180^{\circ}$, or make a $\mathrm{U}$ turn, at some distance from the midplane and move back to the edge, where they are dragged into a current sheet.

Very often the leading edge of the ICME (the sheath) is also known to contain a current sheet (Chian and Muñoz, 2011) that most probably represents an inseparable feature of each ICME. During the past decades several authors claimed to have observed a magnetic reconnection at such pre-ICME current sheets (McComas et al., 1994; Gosling et al., 2006; Gosling, 2007; Phan et al., 2011). A key element in understanding these phenomena is the combination of observational data and theoretical models to reproduce the characteristics of these complex systems and then to derive the fundamental parameters that govern this process. Direct measurements of the magnetic field in the current sheets occurring during the ICME passage can be complemented by measurements of the characteristics of particles and waves outflowing from the current sheet exhausts in order to build a full picture of the processes associated with ICMEs.

High-energy particles are shown to carry fingerprints of the magnetic field topology of the current sheet into which they are dragged and in which they are, consequently, accelerated until they gain the energy sufficient to break from the current sheet (see the review by Zharkova et al., 2011, and references therein). Particle-energy gains depend on a combination of the transverse and guiding magnetic components (Zharkova and Gordovskyy, 2004, 2005; Zharkova and Agapitov, 2009). These components also define the particle pitch angle distribution across the current sheet and the electric field (Siversky and Zharkova, 2009b; Zharkova et al., 2011) induced by the ambient plasma feedback to the separation of accelerated electrons and ions. During their passage across the current sheet, the ion velocities follow the profile of the polarisation electron field very closely. Its skewness to one of the two semiplanes with respect to the current sheet midplane is defined by the ratio of the magnitudes of the magnetic field (transverse and guiding) components, reflecting a domination of either one or another components (Zharkova et al., 2011; Zharkova and Khabarova, 2012).

As shown by PIC simulations applied to the HCS (Zharkova and Khabarova, 2012), the observations of energetic particles in the HCS can be useful in deriving the magnetic field topology responsible for solar-wind particle energisation and the physical conditions responsible for the observed distributions of energetic particles in energy, pitch angles and space.

In particular, the model predicts the appearance of electron clouds formed by bounced electrons far away from the current sheet $X$ null point and caused by the magnetisation of these particles by the guiding magnetic field. The stronger the guiding field, the further from the $X$ null point bounced electrons are found to turn around and to travel back to the side they were injected from, as was observed in the HCS (Zharkova and Khabarova, 2012) and predicted by the PIC model (Siversky and Zharkova, 2009b). Spectral indices of particle-energy spectra are also found to be defined by distributions of the transverse magnetic field, indicating that these indices uniquely define the variations in the transverse magnetic field along the current sheet (Zharkova and Gordovskyy, 2005; Zharkova and Agapitov, 2009).

Hence, the measurements of particle characteristics combined with the measurements of the interplanetary magnetic field in the HCS and in the vicinity of ICMEs will help to understand the conditions of magnetic reconnection events better and to anticipate the arrival of energetic particles after passing these current sheets. This motivated us to explore the parameters of the energetic particles of the fast solar wind during their passage through an HCS with a single boundary or an ICME front with a current sheet and to investigate the possibility of these particles gaining additional energy from the magnetic field topologies of interplanetary or ICME magnetic fields undergoing magnetic reconnection. Understanding the processes leading to additional energisation of the fast solar-wind particles will be useful in defining their effects on space weather, in general, and on communication satellite safety, in particular.

The summary of observations is presented in Sect. 2, the governing model and its results are discussed in Sect. 3, and the comparison of the results of the simulation with observations is discussed in Sect. 4, with conclusions drawn in Sect. 5 .

\section{Summary of observations}

\subsection{Dynamics of energetic particles in the vicinity of the heliospheric current sheet}

The HCS (or sector boundary) is usually associated with multiple crossings (marked by a change in the sign of magnetic field components), reflecting the flapping and waving 
a)

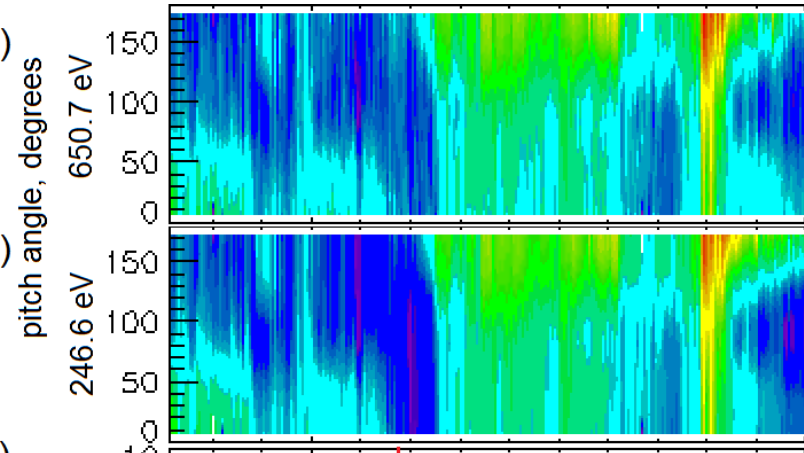

c)

d)

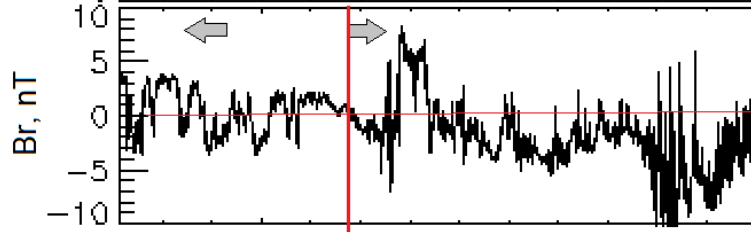

e)

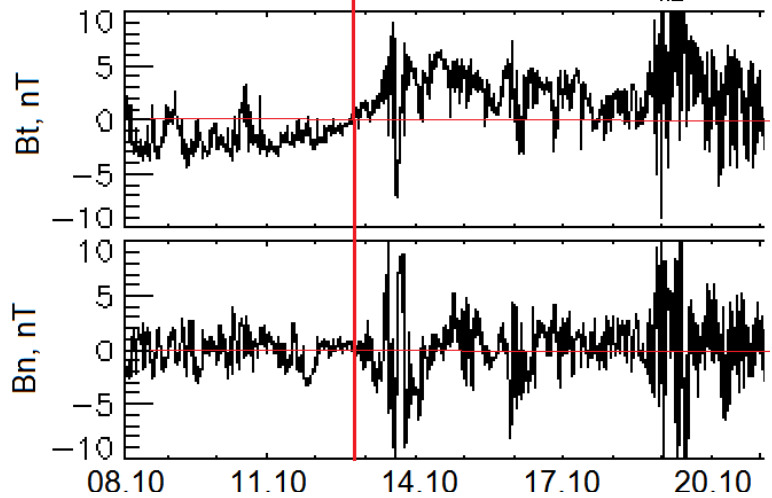

Figure 1. Suprathermal electron pitch angle distribution at $650.7 \mathrm{eV}$ (a) and $246.6 \mathrm{eV}$ (b) compared with the three IMF components $(\mathbf{c}, \mathbf{d}, \mathbf{e})$ measured by STEREO-A in the RTN (Radial Tangential Normal) coordinate system.

HCS and comprising a number of secondary thinner current sheets. However, it was possible to find a rare case of the sector boundary with a relatively clear single boundary crossing that occurred from 9 to 13 October 2007, measured by the Solar TErrestrial RElations Observatory Ahead of the Earth orbit (STEREO-A) spacecraft shown in Figs. 1-3. The HCS crossing is represented by the change in the IMF sign from positive to negative (see Fig. 1c-e). The process of the HCS crossing at $1 \mathrm{AU}$ usually lasts for several days (Khabarova and Zastenker, 2011).

The main HCS crossing occurred on the 12 October 2007, indicated by the red vertical line in Figs. 1-2, in accordance with crossings of the zero line in the IMF components shown in Fig. 1c, $d$ and e. This crossing was accompanied by a large decrease in the IMF strength (Figs. 2d and 3d) and a sharp change in the IMF azimuthal (clock) angle $\phi$ (see Fig. 3e). It is remarkable that the area of sharp changes in the direction of motion for the suprathermal electrons, seen in Fig. 1a and $\mathrm{b}$ as a wide section dominated by green and yellow, at pitch angles $>100^{\circ}$ is shifted from the main HCS crossing indicated by the signatures in the IMF (see Fig. 1c-e). Such shifts of electron $U$ turns have represented a big puzzle for HCS identification for a long time until they were recently explained by the passage of bounced electrons through the reconnecting HCS with a strong guiding field (Zharkova and Khabarova, 2012). We will discuss this phenomenon further in Sect. 3.3 after describing in more detail the simulation of particle motion in a current sheet.

As mentioned above, the boundary crossing in this event was not fast, and there was a wide interface region, predominantly in the positive direction, full of the secondary current sheets observed after the first change in the IMF vector. This interface region is indicated by the two horizontal grey arrows in Fig. 1c-e and 2 around the red vertical line, corresponding to the HCS crossing. Magnetic islands separated by small-scale current sheets effectively scatter electrons inside this region, which looks like a prolonged heat flux dropout in suprathermal electron flux histograms (the blue areas from 9 to 12 October 2007 in Fig. 1a-b). This crossing was accompanied by the high plasma beta (see Fig. 2c), which is one of the distinct signs of a current sheet occurrence.

During the examined period the solar-wind velocity was rather low (as shown in Fig. 2a). The high-speed stream approached the spacecraft several days later, so this was a clean HCS crossing not contaminated by the corotating interacting region (CIR). The slow solar-wind interaction with a current sheet is not shown, while we assume it exists since it supplies the particles entering into the HCS from the solar wind. As seen in Fig. 2e, the fluxes of energetic ions in the 0.15$0.6 \mathrm{MeV}$ and $1.8-10 \mathrm{MeV}$ ranges significantly change near the main HCS crossing. However, their maxima correspond to neither the HCS position (shown in Fig. 2 by the red vertical line) nor to the solar-wind density (Fig. 2b) and velocity (Fig. 2a) changes. The best correlation is seen between the flux variations and the plasma beta, which reflects a dominance in this HCS crossing of the plasma pressure over the magnetic pressure (Fig. 2c).

Figures 2 and 3 demonstrate the acceleration of particles at least up to several $\mathrm{MeV}$ for ions and $0.1 \mathrm{MeV}$ for electrons near the HCS. The variations in the ion flux of solar wind in the energy range of up to several $\mathrm{MeV}$ and electron flux of up to $0.4 \mathrm{MeV}$ are shown in Fig. 3a and b, respectively. The IMF strength and the IMF azimuthal angle can be found in Fig. $3 c$ and d. The main HCS crossing and the intermediate zone containing numerous secondary current sheets and magnetic islands are indicated by the red vertical line, as in Fig. 1.

The first distinct pattern in variations in the ion flux is a clear shift of its maximum from the main HCS crossing (sector boundary) further away from this boundary. The second pattern indicates a significant difference between the distributions of electrons and ions, showing the electron flux increase to spread to a much wider area than the ion flux. However, the electron flux density increase is observed to be ap- 


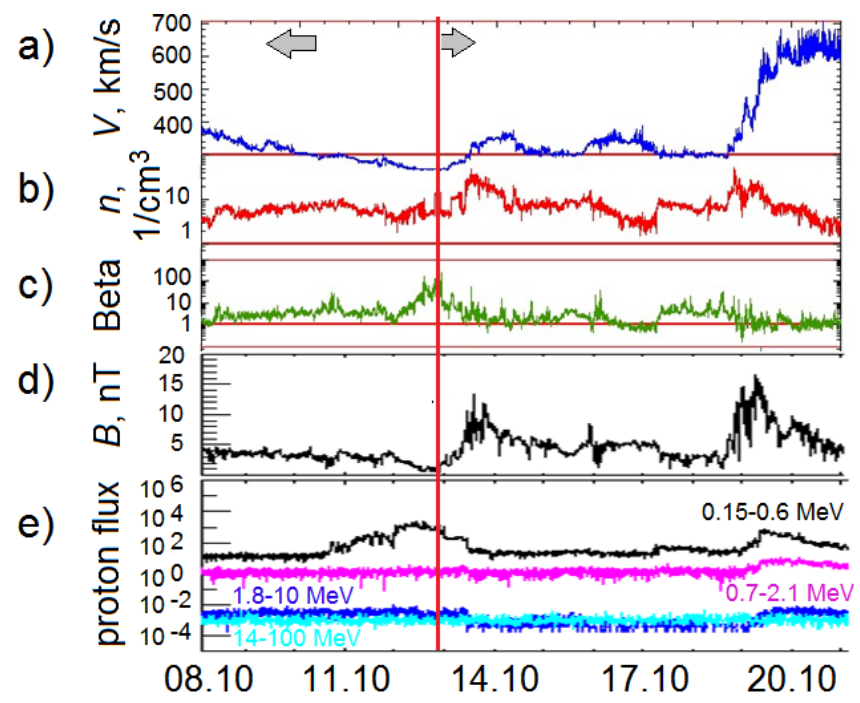

Figure 2. Solar-wind plasma parameters compared with proton flux measured by STEREO-A in different energy channels. (a) The solar-wind speed; (b) plasma density on a logarithmic scale; (c) plasma beta; (d) the IMF strength; (e) and flux of energetic particles (black: 0.15-0.6 MeV; purple: 0.7-2.1 MeV; dark blue: 1.8$10 \mathrm{MeV}$; and light blue: $14-100 \mathrm{MeV}$ ).

proximately symmetrical regarding the main HCS crossing (the purely red area in Fig. 3b).

Hence, in the case of the single HCS crossing presented here, the fluxes of both types of solar wind particles - ions and electrons - are found to have significant topological differences, with the energies of both species increasing significantly to suprathermal level.

\subsection{Tracking energetic particles measured at the leading edges of ICMEs}

Further examples of energetic particles in the solar wind are shown in Fig. 4, obtained from the ACE and Cluster 1 measurements. This ICME event occurred on 21 January 2005 and was considered in detail for Cluster 1 by Chian and Muñoz (2011). We are mainly interested in examining the unusually wide leading ICME edge of the compressed plasma, indicated by the grey arrows in Fig. 4 that shows a comparison of the ACE and Cluster measurements of the total IMF. The event was previously discussed by Chian and Muñoz (2011) on the basis of the Cluster data only. This area of the ICME was full of multiscale current sheets, which look like sharp decreases followed by increases in the magnetic field $\boldsymbol{B}$ and form small-scale magnetic islands that demonstrate the full set of possible signatures of magnetic reconnection in the vicinity of the ICME.

The first sharp increase in the IMF strength in this event corresponds to a shock wave, where the solar-wind velocity changes sharply by up to $1000 \mathrm{~km} \mathrm{~s}^{-2}$ and density experiences an increase of 1 order of magnitude. As seen from

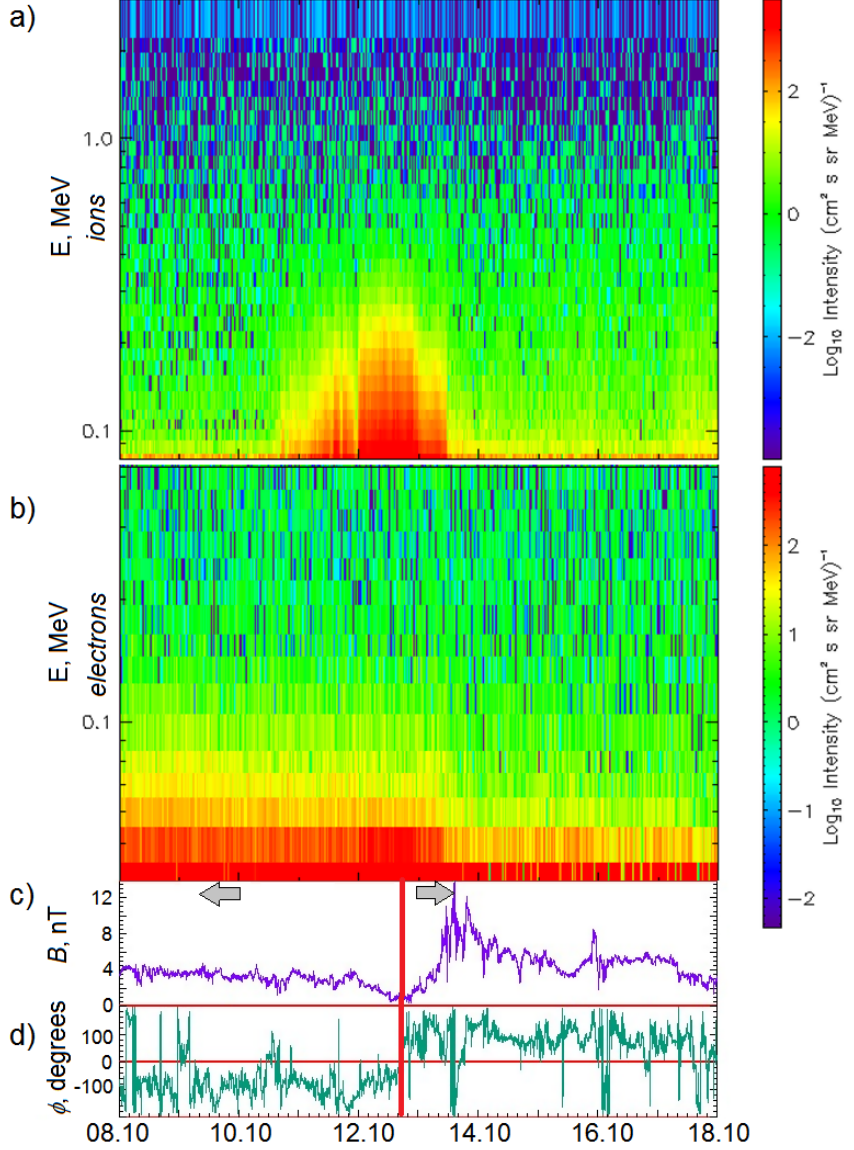

Figure 3. Energy flux of ions (a) and electrons (b) (STEREO-A, spectrograms of energetic particles); the IMF strength (c) and its azimuthal (clock) angle changes (d) during the HCS crossing.

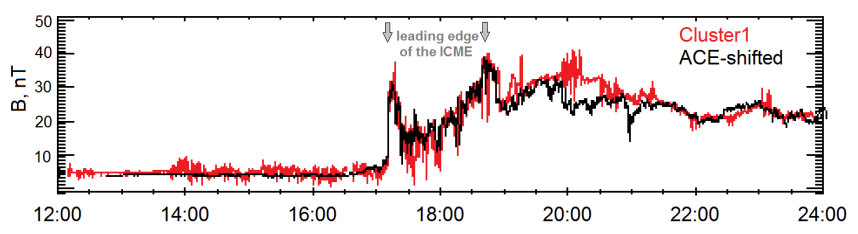

Figure 4. The interplanetary magnetic field strength measured by ACE (black) and Cluster 1 (red) during the passage of the highspeed ICME on 21 January 2005. ACE 16 s resolution data are shifted to the Cluster 1 position. The leading edge region filled with current sheets is indicated by arrows.

Fig. 4, despite a spatial difference between the spacecrafts $\mathrm{ACE}$ and Cluster 1 of $\approx 200 R_{\mathrm{e}}$, the IMF structure inside the leading edge was very stable and changed with distance only inside the magnetic cloud, after the passage of the region under investigation. This means that the current sheets associated with the ICME leading front discussed by Chian and Muñoz (2011) are not local but represent stable structures propagating together with the body of the ICME. 


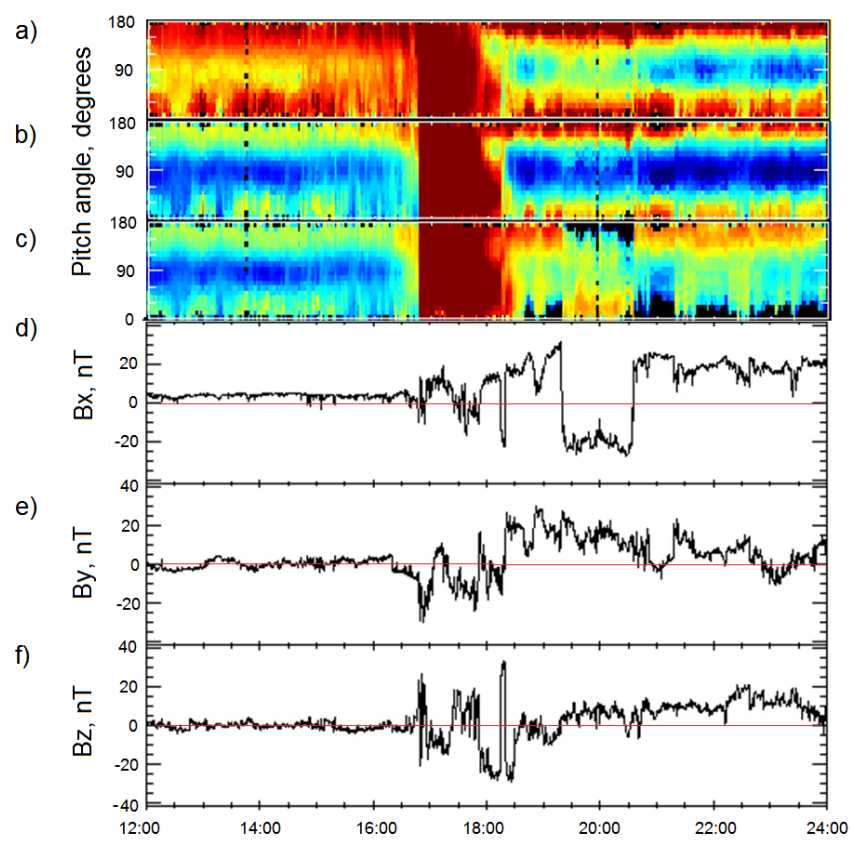

Figure 5. Suprathermal electron pitch angle spectrograms at different energies (a-c) compared with three components of the IMF (df) during the 21 January 2005 event. (a) $519 \mathrm{eV}$ flux, (b) $142 \mathrm{eV}$ flux, (c) $73.3 \mathrm{eV}$ flux, (d, e) in-ecliptic components of the IMF, (f) the vertical component of the IMF in GSE.

Taking into account this fact and using the ACE instrumental possibilities, in addition to facts already known about this event, one can find a few additional features of particle acceleration. Let us show that some of them may be attributed to the current sheets. The pitch angle distribution functions of suprathermal electrons measured by the Solar Wind Electron, Proton, and Alpha Monitor (SWEPAM) onboard of the ACE spacecraft at different energies are compared with the three components of the IMF in Fig. 5. The blue colour in Fig. 5a-c corresponds to low values and the red to high values of the distribution function. Detailed explanations of the data are given at http://www.srl.caltech.edu/ ACE/ASC/DATA/level3/swepam/index.html.

Again, similarly to the ion flux in the HCS, an increase in high-energy particle flux occurs earlier than the ICMEassociated shock comes (seen in the far right corner of each plot in Fig. 5a); this cannot, then, be responsible for this particle acceleration. Also, there is a sharp change in the direction of motion by suprathermal electrons observed at the moment of the shock crossing and after the passage of the leading edge, indicated by grey arrows in Fig. 4. The fluxes of all energies are increased inside the investigated area (wide red section in Fig. 5a-c), which means that the acceleration of particles occurs in this region. It is easy to see multiple crossings of the neutral lines (current sheets) during this period. The zero line is represented in red in Fig. $5 \mathrm{~d}-\mathrm{f}$ in order to indicate such crossings.

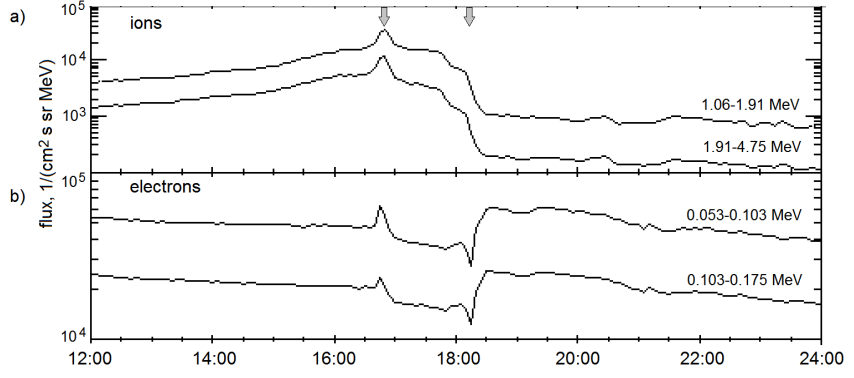

Figure 6. Flux of energetic particles of different energies from ACE EPAM (Advanced Composition Explorer Energetic Proton and Alpha Monitor), 5 min resolution data: (a) ions, (b) electrons. The leading edge of the ICME is indicated by arrows.

The most remarkable feature in this particular ICME is the presence of the bidirectional "strahl" electrons located at the both sides of the ICME front. The strahls look like the two horizontal ribbons seen in the pitch angle spectrograms along 0 and $180^{\circ}$ (Fig. 5a-c). They are particularly pronounced in the highest-energy channel (Fig. 5a). Strahls are known to be suprathermal electron beams, travelling along the magnetic field direction. Their origin is still unclear; however, their properties have been studied for many years (Gosling, 1990; Gosling et al., 2002). It is known that strahls predominantly appear anti-sunward in regular solar wind, although near ICMEs counterstreaming strahls may be also observed. We present their proposed interpretation below in Sect. 3.3.

It can be observed that the accelerated ions and electrons of high (MeV-range) energies occurred several hours earlier than the ICME's leading front reached the Earth orbit, as seen in Fig. 6. Acceleration at the shock is seen as the first sharp increase in both ion and electron flux of different energies. The leading edge of the ICME, indicated by the arrows, is clearly reflected in the ion and electron flux changes.

The local increase in both ion (Fig. 6a) and electron (Fig. 6b) fluxes, corresponding to the shock arrival (the left arrow in Fig. 6), indicates the point at which particles experience reflection and acceleration. This mechanism is responsible for a gradual increase in ions and electrons fluxes, forestalling the ICME arrival. Electrons, being lighter and faster, go further from the source of acceleration (ICME front) than ions. A local increase in the ion flux is observed approximately from 15:00 UT (the foreshock area) to 18:30 UT, corresponding to the border of the leading edge. As a result, the temporal profiles of ion and electron fluxes observed in the vicinity of the ICME become essentially different, resembling the ones occurring in the solar-wind particles during their crossing of the HCS, as shown Fig. 3.

It is remarkable to note that the flux of suprathermal ions does not fall immediately after the ICME's shock crossing (as one would expect if the particles are to be accelerated by this shock); it fell only after the passage of the current sheet area in the front of the ICME. This effect can not be simply 


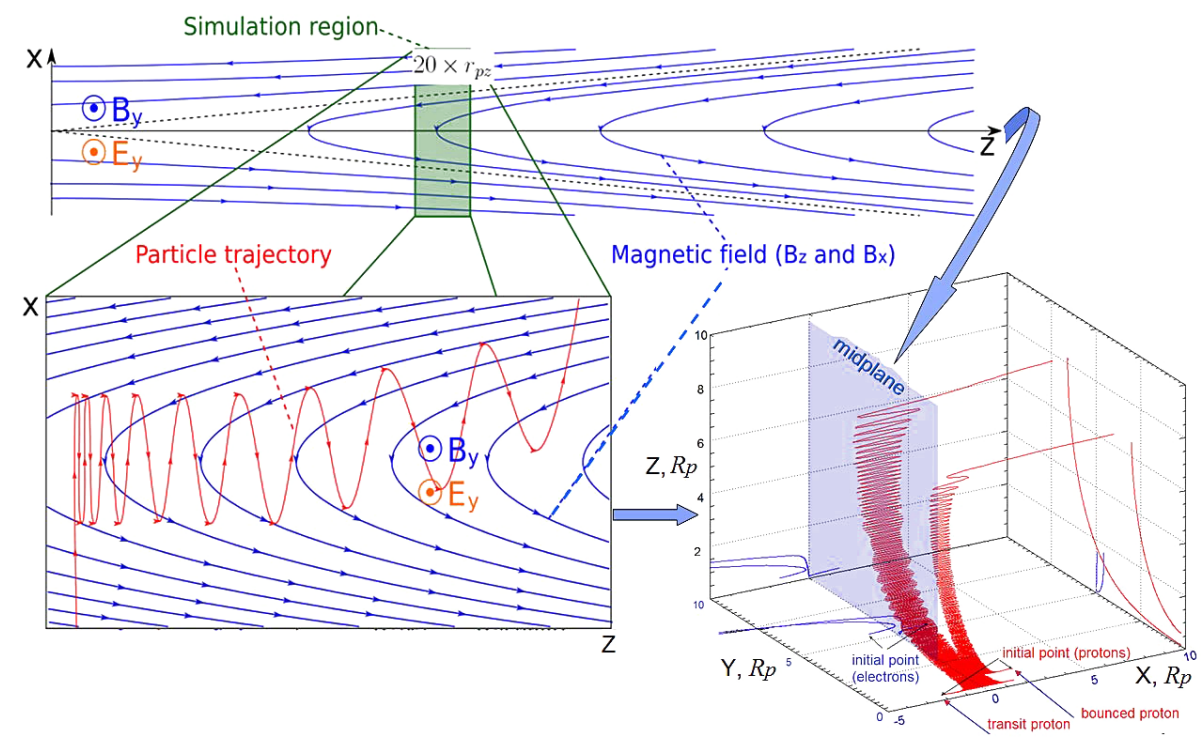

Figure 7. The PIC simulation region (with a close-up at the bottom left) with respect to a whole current sheet (courtesy of Siversky and Zharkova, 2009b). The current sheet simulation plane is the $X Z$ plane; the plane $X=0$ is the current sheet midplane (indicated by blue shading in the bottom-right close-up). $\boldsymbol{E}_{y}$ is the electric field brought into a diffusion region by a magnetic field reconnection (a reconnection electric field); $\boldsymbol{B}_{y}$ is a guiding magnetic field component. The boundaries of the rectangle are accepted to be transparent to particle motion and the periodic boundary conditions used (see the text for details). The close-up at the bottom right shows trajectories of transit and bounced protons (red lines) and electrons (blue lines).

explained by the enhanced solar-wind density because the parameter, characterising the low-energy solar wind, does decrease simultaneously with the ion flux, as shown in Fig. 6a. Hence, this effect is not a result of the flow's topology. This explicitly indicates the topological difference in the motion and acceleration of particles with the opposite charges, which is the most important property of particle acceleration in a reconnecting current sheet (Zharkova and Gordovskyy, 2004). One can see a noticeable difference in the variations in ions and electrons: the electron flux increases at the same time as the ion flux drops, i.e. electrons anticorrelate, and the centre of this inversion is definitely the leading edge of the ICME, where a current sheet is likely to occur.

\section{Snapshots of particle acceleration models in reconnecting current sheets}

\subsection{Magnetic field topology}

The acceleration time during particle motion inside a current sheet is estimated to be of the order of $10^{-6} \mathrm{~s}$ for the electrons and $10^{-3} \mathrm{~s}$ for the protons (Zharkova and Gordovskyy, 2004). This time is much shorter than the time of the magnetic field reconstruction during a reconnection (Priest and Forbes, 2000); thus, the background magnetic field can be assumed to be stationary during the whole simulation.

Also, from the previous test particle simulations in the solar corona, we conclude that the travel distances of acceler- ating particles along the reconnecting current sheet (RCS) are of the order of $10 \mathrm{~km}=10 R_{\mathrm{p}}$ at most (for the protons in the corona) (Zharkova and Agapitov, 2009), a value obtained by applying the periodic boundary conditions along $z$ direction. On the other hand, it is evident that this travel distance is much shorter than the length scale of the magnetic field variation along the current sheet. In addition, as is generally accepted, we suppose that the magnetic field variation across the current sheet has a much shorter length scale than its variation along the current sheet of length $a$ in the direction $z$ and of $b$ in the direction of $y$, e.g. $d \ll a, b$.

Let us consider a coordinate system related to the current sheet midplane, which hypothetically can be oriented in any direction in the solar wind. Our simulation domain is a small part of the reconnecting current sheet (see Fig. 7 for the model cartoon), which is large enough to contain the full trajectories of accelerated particles for the periodic boundary conditions (see Sect. 3.2.1). All the three components of the background magnetic field are considered; the components are assumed to be stationary and to vary inside this domain only in the $x$ direction across the RCS. The main magnetic field component, $\boldsymbol{B}_{z}$, corresponding in most cases to the component $\boldsymbol{B}_{x}$ in system Geocentric Solar Ecliptic coordinate system (GSE) used for the interplanetary magnetic field (IMF), depends on the simulation coordinate $x$ as follows:

$\boldsymbol{B}_{z}(x)=-\boldsymbol{B}_{z 0} \tanh \left(\frac{x}{L_{x}}\right)$. 
The transverse magnetic field component $\boldsymbol{B}_{x}$ corresponding to $\boldsymbol{B}_{y}$ (in GSE) is assumed constant inside the simulation domain, e.g.

$\boldsymbol{B}_{x}=-\boldsymbol{B}_{x 0}$.

The guiding (out-of-plane) magnetic field $\boldsymbol{B}_{y}$ corresponding to the $\boldsymbol{B}_{z}$ component of the IMF (GSE system) is assumed to be maximal in the midplane and to vanish outside the RCS as follows:

$\boldsymbol{B}_{y}(x)=\boldsymbol{B}_{y 0} \operatorname{sech}\left(\frac{x}{L_{x}}\right)$.

Note that for $\boldsymbol{B}_{y 0}=0$ the configuration corresponds to the Harris sheet equilibrium and for $\boldsymbol{B}_{y 0}=\boldsymbol{B}_{z 0}$ the equilibrium becomes force-free.

The inflow of plasma into an RCS combined with the condition of the frozen-in magnetic field leads to the induction of the drifted (out-of-plane) electric field $\boldsymbol{E}_{y}$. In order to provide the inflow of plasma in our simulation domain, we set up a background electric field that drifted in with velocity $V_{\text {in }}$ by a magnetic diffusion process (Priest and Forbes, 2000).

$\boldsymbol{E}_{y}=\boldsymbol{E}_{y 0}=\boldsymbol{B}_{z 0} V_{\text {in }}$,

where $V_{\text {in }}$ is the inflow velocity, which is about 0.1 times the Alfvén speed. It is usually accepted as being equal to the thermal velocity of the ambient plasma (Zharkova and Gordovskyy, 2004). The magnetic field gradient of the main magnetic field component $\boldsymbol{B}_{z}$ across the current sheet thickness (along the $x$ axis) is ignored (Zharkova and Gordovskyy, 2004).

\subsection{Description of the calculations}

\subsubsection{Simulation method}

We used the 2D3V PIC simulation code developed by Verboncoeur and Gladd (1995). The PIC method is based on the equation of motion for plasma particles plus the two Maxwell equations for the electric and magnetic field induced in the ambient plasma by accelerated particle themselves (Siversky and Zharkova, 2009b).

We define the 3-D magnetic field topology as above and do the double integration of the differential equations to obtain, first, the 3-D distribution of particle velocities and then the 2-D distribution of particle locations. In these 2-D simulations the $y$ dimension is chosen to be invariant. Since we solve a system of partial differential equations in a limited simulation region, we consider the periodic boundary conditions in the $z$ direction, similar to other studies of such a problem (Makov and Payne, 1995; Verboncoeur and Gladd, 1995; Zeiler et al., 2002), so that a particle that leaves the system through the right or left boundary (see Fig. 7 for the model cartoon) appears on the opposite boundary. This eliminates the need to make the system very long in order to handle the whole particle trajectory from entering to ejection, as defined by Zharkova and Agapitov (2009).
For the simulations we used the current sheet halfthickness, $d$, to be equal to the gyroradius of protons, $R$ (or $1 \mathrm{~m}$ for the coronal conditions), so that $d=2 R_{\mathrm{p}}$, while the width of the whole simulation region along $x$ is chosen to be $20 \times R_{\mathrm{p}}$ (in some cases $50 \times R_{\mathrm{p}}$ ) in order to avoid any influence of the boundaries on the particles inside the RCS. Plasma is continuously injected from the $x= \pm 10 \times R_{\mathrm{p}}$ sides of the simulation region at a rate of $n \boldsymbol{E}_{y 0} / \boldsymbol{B}_{z 0}$.

In order to avoid numerical instabilities in the PIC method, the following constraints need to be satisfied:

$c \Delta t<\Delta \xi$,

$\Delta t<0.2 \omega_{\mathrm{pe}}^{-1}$,

$\Delta \xi<\lambda_{\mathrm{D}}$

where $\Delta t$ is the time step, $\Delta \xi$ is the grid step in any direction, $c$ is the speed of light, $\omega_{\mathrm{pe}}=\left(4 \pi n e^{2} / m_{\mathrm{e}}\right)^{1 / 2}$ is the electron plasma frequency and $\lambda_{\mathrm{D}}=\left(k T / 4 \pi n e^{2}\right)^{1 / 2}$ is the Debye length. We use the plasma density inside a current sheet of $n=10 \cdot 10^{6} \mathrm{~m}^{-3}$ relevant to the heliosphere. Also, the proton-to-electron mass ratio is reduced to $m_{\mathrm{p}} / m_{\mathrm{e}}=100$ in order to keep the proton acceleration time within reasonable computational limits. The spatial simulation grid has 100 cells in the $z$ direction and 100 cells in the $x$ direction, with $\Delta z=\lambda_{\mathrm{D}}$ and $\Delta x=\lambda_{\mathrm{D}} / 5$ and 100 particles per cell on average. The time step is $6 \cdot 10^{-10} \mathrm{~s}$.

\subsubsection{Scaling the simulation region to heliospheric conditions}

For the simulation we used the following values for the current sheet parameters relevant to the solar corona (Siversky and Zharkova, 2009b): the main component of the magnetic field $\boldsymbol{B}_{z 0}=10^{-3} \mathrm{~T}$, the current sheet half-thickness $L_{x}=$ $1 \mathrm{~m}$ that corresponds to the gyroradius of protons for a magnetic field of $\boldsymbol{B}=10^{-2} \mathrm{~T}$ and the drifted electric field $\boldsymbol{E}_{y 0}=$ $250 \mathrm{~V} \mathrm{~m}^{-1}$. The magnitudes of the transverse magnetic field, $\boldsymbol{B}_{x 0}$ varied from $10^{-4} \boldsymbol{B}_{z}$ to $\boldsymbol{B}_{z}$ and, for the guiding magnetic field, the $\boldsymbol{B}_{y 0}$ parameter was used as $\boldsymbol{B}_{y}=(0.05-0.1) \boldsymbol{B}_{z}$ (for a weak guiding field approach) and $\boldsymbol{B}_{y}=(0.5-1) \boldsymbol{B}_{z}$ (for a strong guiding field).

In order to adopt the simulation region to the heliospheric current sheets, we applied a scaling of the simulation region by the gyroradius of protons, similar to the one we applied earlier (Zharkova and Khabarova, 2012).

For the IMF magnetic field variations of $10^{-8}-10^{-9} \mathrm{~T}$, as measured at $1 \mathrm{AU}$ or even lower (see for example, the event shown in Figs. $1-3 ; \mathrm{V}=270 \mathrm{~km} \mathrm{~s}^{-1}, \boldsymbol{B}=0.3 \mathrm{nT}$ ), the gyroradius of proton, $R_{\mathrm{p}}$, reaches $R_{\mathrm{p}}=(1-10) \cdot 10^{6} \mathrm{~m}$ or $R_{\mathrm{p}}=$ $(1-10) \cdot 10^{3} \mathrm{~km}$, which is comparable with the size of a single $\mathrm{HCS}$ at $1 \mathrm{AU}\left(10^{2}-10^{4} \mathrm{~km}\right)$. For the physical conditions in the heliosphere, where the magnetic field is reduced by more than 7 orders of magnitude compared to the corona, the reconnecting electric field, which causes particle acceleration, is also reduced (see Eq. 4). Thus, the simulation region used 
for the coronal parameters for $20 R_{\mathrm{p}}=20 \mathrm{~m}$ can be rescaled to the heliosphere $\left(20 R_{\mathrm{p}}=20 \times(1-10) \cdot 10^{4} \mathrm{~km}\right)$, covering the region up to $200000 \mathrm{~km}$ around the sector boundary (HCS) to each side of the midplane.

This means that, in real PIC simulations for the heliospheric conditions, one can run the calculation for magnetic and electric field parameters relevant to the coronal conditions while using the densities relevant to the heliosphere. Then the results of simulations can be rescaled onto a new gyroradius of protons for the magnetic field magnitude relevant to the heliosphere, where magnetic field components are reduced by 6-7 orders of magnitude, as is the reconnection electric field (as per Eq. 4). Since the energy gains by each kind of particles are mostly proportional to the ratios of the magnetic field components (Siversky and Zharkova, 2009b, see formulae 6 and 7 and their discussion), they are not affected by a magnetic field magnitude change. This is valid for most cases, apart from the energy of strongly magnetised electrons when their energy gains are reduced accordingly (e.g. bounced electrons in a strong guiding field approach).

\subsection{Results of simulations}

In Fig. 8 we plotted the results of the two PIC simulations carried out for a weak guiding field (Fig. 8a: protons; b: electrons) and for a strong guiding field (Fig. 8c: protons; d: electrons).

There are a few important outcomes of the particle acceleration simulation in a reconnecting current sheet. The first one indicates that, for the magnetic topologies with a moderateto-strong guiding field, there is a strong separation of electron trajectories into one semiplane (say with $X>0$ ) and proton trajectories into the opposite one (with $X<0$ ), as shown in Fig. $8 \mathrm{c}$ and $\mathrm{d}$ for PIC simulations or in the bottom-right closeup of Fig. 7 obtained with the test particle approach for the given magnetic field topology.

In the case of a weaker guiding field (Fig. 8a and b), there is a little or no separation of protons (a) and electrons (b); the protons are ejected slightly more to the negative semiplane but some are still ejected to the positive one. Electrons are found cycling around the midplane because they cannot gain sufficient energy to break from the electrostatic force of protons being accelerated in the midplane. As a result, electrons create a cloud, which exists for as long as protons are present in the midplane, and then they follow the protons and become ejected to the same semiplane ( $X<0$ in Fig. $8 \mathrm{a}$ and b).

The second outcome, related to the first, reveals the origin of two beams of the same charge: (1) the transit particles, which enter from the side opposite to the one from which they are to be ejected (for example, protons from the side $X<0$ and electrons from $X>0$ in the right-hand close-up in Figs. 7 and 8) and (2) the bounced particles entering the RCS from the same side from which they will be ejected (protons from the side $X>0$ and electrons from the side $X<0$ ).
The transit particles start gaining their energy from a reconnecting electric field $\boldsymbol{E}_{y}$, immediately after they are dragged into the diffusion region, while they still travel to the current sheet midplane, where the bulk of their energy is to be gained. Hence, their gyration around the midplane has large radii and they gain much more energy than the bounced electrons, which need to work against the magnetic field topology in order to arrive at the midplane, the only place in an RCS where the particles can be accelerated. Hence, the gyro radii of bounced particles become much smaller than those of the transit particles, and they gyrate closer to the midplane in the case of a weaker guiding field, thus gaining less energy (see Fig. 8a for protons and $\mathrm{b}$ for electrons).

In the case of a stronger guiding field, the transit particles gain more energy than in a weaker one (Fig. 8c and d), while the bounced electrons cannot even reach the midplane (Fig. 8d). These bounced electrons are turned around by the magnetic field back in the direction they came from; they then form a shoe-like density distribution, shown in Fig. 8d, or even take on a medallion-type shape (Zharkova and Khabarova, 2012). The distance after which the bounced electrons turn around is inversely proportional to the guiding field magnitude, which was also confirmed earlier by the observation of electron pitch angles for the three cases of the measured magnetic field topologies in the HCS crossings summarised in Table 1 of the paper by Zharkova and Khabarova (2012).

The third outcome is related to the ambient plasma feedback to a presence of accelerated electrons and protons, whose separation towards the midplane induces a strong polarisation electric field across the current sheet (in the directions $X$ and $Z$ ) caused by the particle separation towards the midplane. Some examples of this polarisation electric field $\widetilde{\boldsymbol{E}}_{x}$ are shown in Fig. 9 for the magnetic field topology of the heliosphere for weaker and stronger guiding fields. The magnitude of the polarisation electric field $\left(\widetilde{\boldsymbol{E}}_{x}\right)$ exceeds the original reconnection electric field $\boldsymbol{E}_{y}$ by 1-2 orders of magnitude. This electric field defines the direction of motion of the protons and ions passing through any RCS, as discussed in the next section.

\section{Comparing observations with the simulation results}

\subsection{Particles crossing the HCS}

The main HCS crossing and the intermediate zone containing numerous secondary current sheets and magnetic islands are indicated by the red vertical line, as in Fig. 1c-e. Figures 2 and 3 demonstrate acceleration in the vicinity of the HCS of solar-wind electrons to $100 \mathrm{keV}$ and of ions to several MeV. The ion velocity profile (Fig. 2) has a minimum before entering into the HCS, followed by its maximum at some distance after passing through the HCS. Using the other STEREO data, it is possible to find a number of similar cases with a 
a)

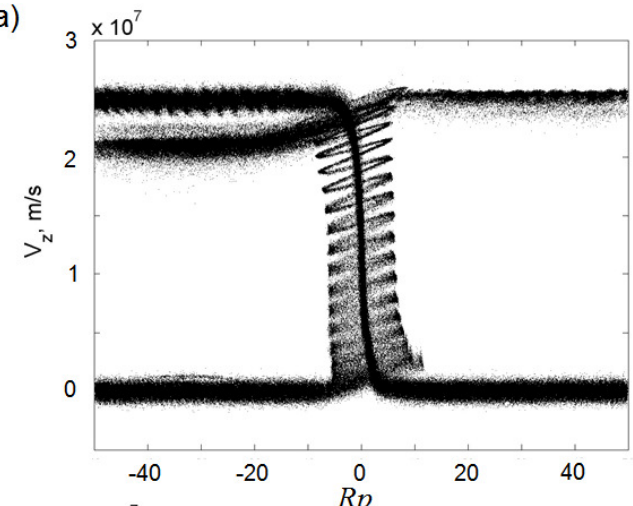

b)

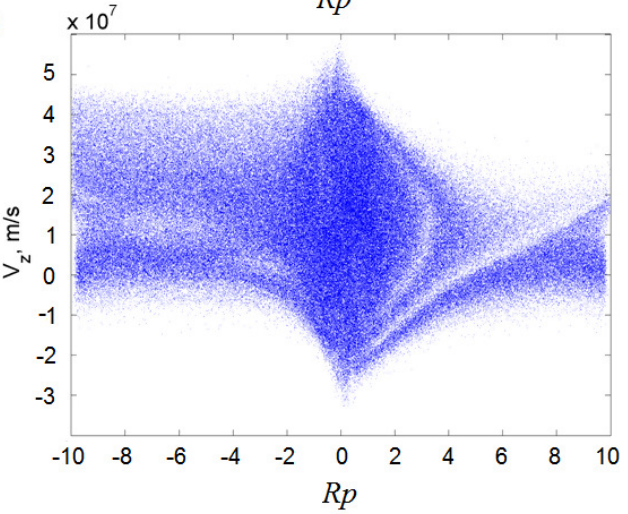

c)

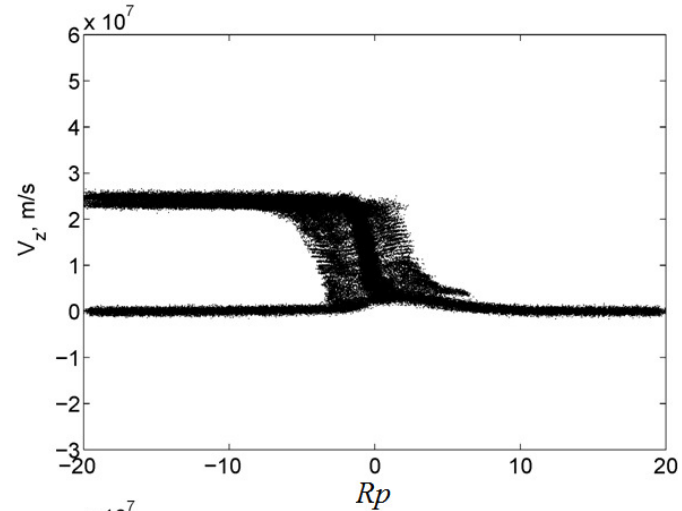

d)

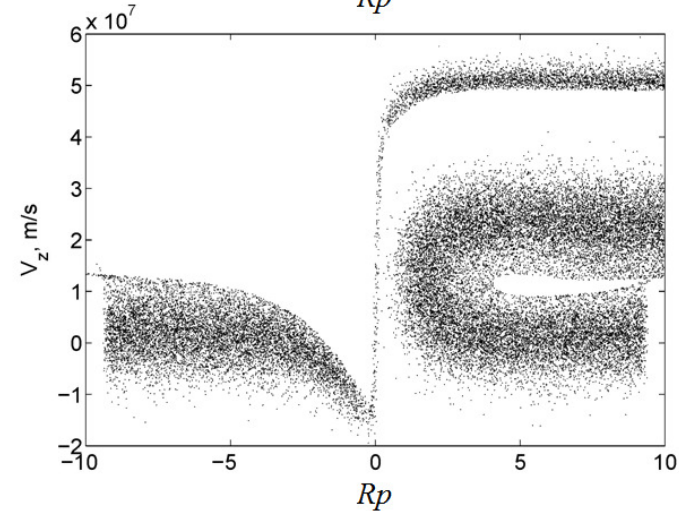

Figure 8. Particle distributions simulated with PIC for the magnetic topology (low magnetic field, low plasma density): (a) distribution of protons (transit and bounced) for a weak guiding field $\left(\boldsymbol{B}_{y}=0.01 \boldsymbol{B}_{0}, \boldsymbol{B}_{0}=10^{-8} \mathrm{~T}\right)$, showing protons ejected to both sides of the midplane; (b) distribution of electrons for the same weak guiding field as in (a), forming an electron cloud about the midplane (transit electrons), with bounced electrons ejected to the same side as protons in (a); (c) distribution of protons for a strong guiding field $\left(\boldsymbol{B}_{y}=0.5 \boldsymbol{B}_{0}, \boldsymbol{B}_{0}=10^{-8} \mathrm{~T}\right)$ with ejection to the negative semiplane $(X<0)$ (full separation from electrons); (d) distribution of electrons for the same guiding field as in (c), with electrons being ejected to the positive semiplane $(X>0)$ as a high-energy stream (transit electrons) and as a shoe-like low-energy flux (bounced electrons).

single boundary crossing, a few of which were reported by Zharkova and Khabarova (2012). The electron pitch angle measurements shown in Fig. 1a and $\mathrm{b}$ also demonstrate the electrons turning their direction of motion by $180^{\circ}$ at some distance before the sector boundary crossing.

This summary of the particle versus magnetic field measurements allows us to conclude that it is difficult to find any structures in the solar wind potentially responsible for these specific particle acceleration profiles, except for the fact that they pass through the HCS. Thus it is logical to assume that some local re-acceleration can occur at the HCS, and this can be the case in the HCS undergoing a magnetic reconnection.

In the case of a reconnecting HCS, one can involve the results of simulations shown in Sect. 3, where the magnetic field zero is reached at a current sheet midplane $(X=0)$. Then it becomes more evident how the electrons and protons can reach the energies reported in observations after they pass a current sheet. This happens because they gain energy there up to a few $\mathrm{MeV}$ for protons and $100 \mathrm{keV}$ for transit electrons (see model results for higher-energy protons and electrons in Fig. 8a, c and d). This model simulation explains the results reported in Figs. 2 and 3.

By contrast, for the low magnetic field of the HCS and the higher guiding field, the bounced electrons cannot approach the midplane of the HCS and are turned around by the magnetic configuration, making them move back to the point at which they were dragged into the HCS, as shown in Fig. 8d. This explains very naturally the variations in the pitch angle distribution of electrons shown in Fig. 1a and b and the dependence of the distance at which this turn occurs on the magnitude of the guiding magnetic field, as reported in Table 1 (Zharkova and Khabarova, 2012). Hence, by measuring this distance one can derive the simulated ratio between the guiding field $\boldsymbol{B}_{y}$ and the main magnetic field component $\boldsymbol{B}_{z}$, compare it with the measured one and explain the $\mathrm{U}$ turn of solar-wind electrons.

The peculiar profiles of the ion flux velocities with a minimum before and a maximum after the HCS can be naturally explained by the polarisation electric field induced by the separation of accelerated particles of the opposite charge shown in Fig. 8c and d-the ions simply follow this polarisa- 


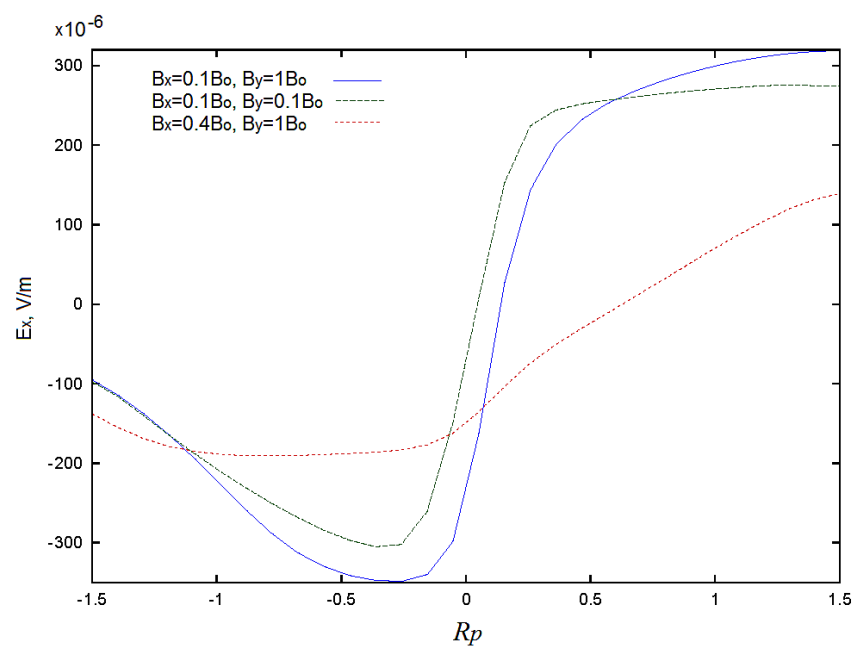

Figure 9. The polarisation (Hall) electric field $\widetilde{\boldsymbol{E}}_{x}$ versus the distance $X$ from the midplane (measured in the units of $R_{\mathrm{p}}$ ) induced by accelerated particles at their separation in the midplane according to different values of $\boldsymbol{B}_{x 0}$ and $\boldsymbol{B}_{y 0}$ for the magnitude of $\boldsymbol{B}_{z 0}=10^{-9} \mathrm{~T}$.

tion electric field during their passage across the HCS. From a profile of the ion velocity across the HCS, one can estimate the polarisation field magnitude using the ratio of the guiding $\boldsymbol{B}_{y}$ and transverse $\boldsymbol{B}_{x}$ magnetic field components.

Of course, everything said above is valid for a single current sheet as modelled. However, the additional scenarios of particle acceleration can occur in magnetic islands formed in a reconnecting current sheet, and this can be valid for the HCS (Drake et al., 2009; Neergaard Parker et al., 2014; Zank, 2014). Additional mechanisms of the acceleration of particles in the vicinity of the merging magnetic islands observed near the HCS and separated by secondary current sheets (Zank et al., 2014) may explain the existence of a wide area of the HCS filled with energetic particles. If so, the particles can be initially accelerated by the reconnecting electric field of the current sheet discussed here and by Zharkova and Khabarova (2012) and, then, with the evolution of the current sheet caused by tearing instability and the formation of a number of magnetic islands as a result (Drake et al., 2006), they can become trapped in closely located magnetic islands, leading to their secondary acceleration (Drake et al., 2006, 2009; Zank et al., 2014).

There is another application of the obtained results for the particles passing through the HCS. It is commonly accepted that, in the absence of SEP events, CIRs are the main source of suprathermal particles in the solar wind (Gómez-Herrero et al., 2011). This was not the case in the observations presented in Figs. 1-3; the CIR was very far from the HCS and it came too late to explain the observed effects. It is important to note that, in most cases, the HCS is crossed before the CIR occurrence at $1 \mathrm{AU}$, i.e. additional acceleration of the solar-wind particles actually takes place in the reconnecting heliospheric current sheet but not at the CIR itself.

\subsection{Particles passing across ICMEs}

In the case of the ICME, additionally to the well-known mechanism of acceleration by shocks, here we suggest particle acceleration by reconnecting current sheets occurring at the leading edge of this propagating structure. The ICMEassociated current sheets seem to be relatively stable structures, as seen from a comparison of the Cluster and ACE measurements, and some features of the energetic particles in their vicinity may be attributed to a magnetic reconnection process. Energetic-particle fluxes demonstrate local enhancements around the area filled with current sheets, typical for the particles' movement through reconnecting current sheets. One of the most interesting features of particle acceleration in a current sheet is the anticorrelation of ion and electron flux and their different pitch angle distributions (separation of ions and electrons into the opposite semiplanes), which is consistent with the PIC results presented here (see the first outcome of simulations in Sect. 3.3).

Similarly to the ion flux in the HCS, an increase in highenergy particle flux up to a few MeV occurs in the ICME before the associated shock that is accompanied by a sharp change in the direction of motion by suprathermal electrons observed at the moment of the shock crossing and after the passage of the leading edge, indicated by the grey arrows in Figs. 4, 6 and seen as the red section in Fig. 5. It can be seen that these energy gains by ions and electrons as well as the change in direction of motion by electrons can be explained by the mechanism of particle acceleration in a reconnecting current sheet in the front of the ICME, in a similar way as was done above for the HCS.

Furthermore, from the profile of ion velocities and from the distance at which the suprathermal electron changes its direction, one can deduce some information about magnetic and electric field components leading to such particle profiles. There is a noticeable difference in the variations in ions and electrons: the ion flux decreases after the leading edge crossing (Fig. 6a), while the electron flux, by contrast, increases before this crossing (Fig. 6b); this can be explained naturally by the separation of particles with opposite charges with respect to the current sheet midplane (similar to the particles shown in Fig. 8c and d and their consequent ejection to the opposite semiplanes (e.g. the opposite sides of the exhausts of a current sheet)).

The most remarkable feature of this particular ICME is the presence of the bidirectional high-energy electrons (strahls) on both sides of the ICME front. The term strahl is used here according to the definition of (Gosling et al., 2004a, b), considering strahls to be the narrowest field-aligned beams. The strahls are seen in the highest-energy channel as the two horizontal ribbons in the pitch angle spectrograms along 0 and $180^{\circ}$ (Fig. 5). Indeed, simultaneous changes in the strahl in- 
tensity at all energies are commonly observed near the HCS and at the leading edges of CIRs and ICMEs (Gosling et al., 2002, 2004b). In the HCS case, they simply travel along the magnetic field lines, predominantly in the anti-sunward direction, but at the ICME edges their symmetric depletions are observed.

The strahls are usually assumed to propagate outward from the corona, i.e. to have a solar origin. An alternative view is to consider strahls as suprathermal electrons accelerated due to the resonant interaction with whistler waves, suggested by Vocks and Mann (2003), although, this theory cannot be used to explain their bidirectionality near ICMEs. This bidirectionality was interpreted as a consequence of the double magnetic connection of ICMEs to the Sun. However, the bidirectional strahls are observed at corotating shocks very far from the Earth, at $5 \mathrm{AU}$ (Gosling et al., 1993), where such a supposition is very doubtful because of the long distance from the Sun; it thus requires some additional suppositions, such as mirroring or similar, which are also difficult to maintain.

Meanwhile, our results on the accelerated electron behaviour near a reconnecting current sheet allow an alternative explanation: electron acceleration to the strahl energies occurs locally due to a magnetic reconnection that appears at the HCS, the leading edges of CIRs and ICMEs as well as at corotating shocks, where current sheets are formed. We suggest that the strahls associated with ICME are generated by the most energetic electrons (transit electrons), which are accelerated in a current sheet at the ICME's front to fairly high energies, as shown in Fig. 8d (upper stream).

Then the dependence of electron trajectories in a current sheet on the magnetic field topology can easily explain the bidirectionality of the ICME-associated strahls because the accelerated electrons have a tendency to move to the opposite sides of the midplane to the diagonal quarters with respect to the planes $Z=0$ and $X=0$. It is obvious that, if not disturbed, such a movement of transit electrons must be bidirectional with respect to the plane $Z=0$, as its direction of motion is dependent on the signs of $\boldsymbol{B}_{x}$ and $\boldsymbol{B}_{y}$. For different signs of $\boldsymbol{B}_{z}$, the magnetic topology will follow either the left- of right-hand rule, leading to the bidirectional move of the energetic transit electrons seen as strahls.

The particle transport problem concerning the time after the electrons are ejected from the current sheets where they are accelerated has not been investigated yet. From the general point of view, one can expect that energetic electrons ejected from the HCS into opposite directions (from and to the Sun) have to undergo some interaction with the ambient plasma. The fraction of electrons which moves towards the Sun, where density increases, will be scattered by the ambient particles and lose their energy in Coulomb collisions and ohmic losses, similar to solar-flare electrons (Siversky and Zharkova, 2009a). The difference is that the initial energy of the solar-wind electrons is well below keV; hence, they will lose this energy rather fast while approaching the Sun. By contrast, the ejected electrons moving from the Sun into the rarifying plasma do not meet many particles, and, thus, they can keep propagating as beams of energetic electrons, or strahls.

The geometry of motion of electrons around the ICME's current sheets is slightly different, since the stronger magnetic field of this current sheet in front of the ICME is perpendicular to the weaker interplanetary magnetic field (IMF). The energetic electrons accelerated in this current sheet keep moving along this stronger magnetic field of the ICME's current sheet as long as this current sheet exists. This is why observers can see energetic electrons, or strahls, for a very long time as two electron streams moving in opposite directions as they are ejected from the current sheet. However, a precise particle transport scenario after their ejection from the HCS or the ICME's front current sheet can be only derived from simulations of the process of particle scattering and motion in a given magnetic field topology that will be the subject of a forthcoming paper.

\section{Conclusions}

In this paper we presented the observations of IMF and solarwind particle characteristics in the vicinity of the HCS and ICMEs, which can be naturally explained by the additional acceleration of solar-wind particles occurring during their passage through 3-D reconnecting current sheets of the HCS or the ICME front.

We show that, for the two cases presented, the particle acceleration occurs locally at reconnecting current sheets: either on the background of energetic flux enhancement related to the shock, in the ICME case, or well before the appearance of CIR, in the HCS case. This eliminates the shock or CIR, respectively, as the possible causes of particle acceleration in the observed events, leaving reconnecting current sheets (the HCS and at the one in front of the ICME) as the most likely reason for this acceleration.

The occurrence of current sheets in the interplanetary space (such as the heliospheric current sheet) and at the leading front of the ICME can change the spatial and energy distribution of suprathermal particles gaining an energy of up to several $\mathrm{MeV}$ at the Earth's orbit during their passage through the current sheets; this distribution resembles very closely those seen in the observations.

These observations include the magnitudes of the energy gains by both electrons and ions after their passing through a reconnecting current sheet. It also includes their preferred trajectories uniquely defined by a magnetic field topology of a reconnecting current sheet, leading to the separation of electrons from protons and ions with respect to a current sheet midplane and leading to their preferential ejection from the opposite sides of this current sheet after they gain sufficient energy to break free from the magnetic field of this current sheet. 
This additional acceleration of particles also includes the formation of two beams for each charge: transit particles, e.g. those injected from the side opposite to the one from which they will be ejected, and bounced particles, e.g. those injected from the same side from which they will be ejected. Hence, electrons have transit and bounced electrons and protons and ions have both transit and bounced protons.

Transit particles gain the highest energy for each charge, accounting for most of the energetic particles passing through the HCS or the ICME. Transit electrons are, possibly, seen as strahls in the in situ observations of ICMEs, while bounced electrons reveal a shoe- or medallion-like distribution of their pitch angle in the vicinity of the HCS.

In addition, the feedback of the ambient plasma to the separation of electrons from protons and ions calculated with the PIC approach is expressed in the formation of a polarisation electric field across the current sheet, whose magnitude exceeds that of the original reconnecting current sheet accelerating particles by $1-2$ orders of magnitude. This polarisation electric field defines the velocity profiles of energetic ions during their passage through a current sheet, as seen during the crossing of the HCS discussed here and other crossings, as discussed by Zharkova and Khabarova (2012).

Hence, in summary, the idea of an additional local acceleration of solar-wind particles during their passage through reconnecting current sheets, which occurs in the heliosphere, may explain numerous puzzling effects observed in the vicinity of the HCS and at ICMEs, such as

- the increase in energetic-particle flux in the vicinity of current sheets and, in particular, of the HCS;

- the premature change in the pitch angles of suprathermal electrons before the real HCS crossing, measured by a sign change in the magnetic field;

- the profiles of suprathermal particles observed close to the leading edge of ICMEs;

- the occurrence of bidirectional strahls at the ICME front.

For the interpretation of more complicated observations of multiple current sheets often seen during crossings of the HCS or at the leading edges of ICMEs, there is the need for more sophisticated PIC simulations with multiple reconnection sites, formed by magnetic islands.

Acknowledgements. The authors would like to thank the anonymous referees for their useful and constructive comments from which the paper benefited greatly. The authors also wish to express their thanks to the STEREO team for providing the data on the STEREO IMPACT magnetic field and PLASTIC Data Server http://aten.igpp.ucla.edu/forms/ stereo/level2-plasma-and-magnetic_field.html as well as the STEREO data server http://stereo-ssc.nascom.nasa.gov/data.shtml. O. Khabarova wishes to acknowledge the support of this research by the Russian Fund for Basic Research, grants no. 14-02-00769 and no.14-02-00308.

Topical Editor V. Fedun thanks two anonymous referees for their help in evaluating this paper.

\section{References}

Chian, A. C.-L. and Muñoz, P. R.: Detection of Current Sheets and Magnetic Reconnections at the Turbulent Leading Edge of an Interplanetary Coronal Mass Ejection, Astrophys. J. Lett., 733, L34, doi:10.1088/2041-8205/733/2/L34, 2011.

Crooker, N. U., Forsyth, R., Rees, A., Gosling, J. T., and Kahler, S. W.: Counterstreaming electrons in magnetic clouds near 5 AU, J. Geophys. Res.-Space, 109, A06110, doi:10.1029/2004JA010426, 2004.

Drake, J. F., Swisdak, M., Che, H., and Shay, M. A.: Electron acceleration from contracting magnetic islands during reconnection, Nature, 443, 553-556, doi:10.1038/nature05116, 2006.

Drake, J. F., Swisdak, M., Phan, T. D., Cassak, P. A., Shay, M. A., Lepri, S. T., Lin, R. P., Quataert, E., and Zurbuchen, T. H.: Ion heating resulting from pickup in magnetic reconnection exhausts, J. Geophys. Res.-Space, 114, A05111, doi:10.1029/2008JA013701, 2009.

Gómez-Herrero, R., Malandraki, O., Dresing, N., Kilpua, E., Heber, B., Klassen, A., Müller-Mellin, R., and Wimmer-Schweingruber, R. F.: Spatial and temporal variations of CIRs: Multi-point observations by STEREO, J. Atmos. Sol.-Terr. Phys., 73, 551-565, doi:10.1016/j.jastp.2010.11.017, 2011.

Gosling, J. T.: Coronal mass ejections and magnetic flux ropes in interplanetary space, American Geophysical Union Geophysical Monograph Series, Washington D.C., 58, 343-364, 1990.

Gosling, J. T.: Observations of Magnetic Reconnection in the Turbulent High-Speed Solar Wind, Astrophys. J. Lett., 671, L73L76, doi:10.1086/524842, 2007.

Gosling, J. T.: Magnetic reconnection in the heliosphere: new insights from observations in the solar wind, in: IAU Symposium, edited by Gopalswamy, N. and Webb, D. F., 257, 367-377, doi:10.1017/S1743921309029597, 2009.

Gosling, J. T. and Phan, T. D.: Magnetic Reconnection in the Solar Wind at Current Sheets Associated with Extremely Small Field Shear Angles, Astrophys. J. Lett., 763, L39, doi:10.1088/20418205/763/2/L39, 2013.

Gosling, J. T., Bame, S. J., Feldman, W. C., McComas, D. J., Phillips, J. L., and Goldstein, B. E.: Counterstreaming suprathermal electron events upstream of corotating shocks in the solar wind beyond approximately 2 AU: ULYSSES, Geophys. Res. Lett., 20, 2335-2338, doi:10.1029/93GL02489, 1993.

Gosling, J. T., Skoug, R. M., Feldman, W. C., and McComas, D. J.: Symmetric suprathermal electron depletions on closed field lines in the solar wind, Geophys. Res. Lett., 29, 1573, doi:10.1029/2001GL013949, 2002.

Gosling, J. T., Eriksson, S., and Schwenn, R.: Petschek-type magnetic reconnection exhausts in the solar wind well inside 1 AU: Helios, J. Geophys. Res.-Space, 111, A10102, doi:10.1029/2006JA011863, 2006.

Gosling, J. T., Tian, H., and Phan, T. D.: Pulsed Alfvén Waves in the Solar Wind, Astrophys. J. Lett., 737, L35, doi:10.1088/20418205/737/2/L35, 2011. 
Gosling, J. T., de Koning, C. A., Skoug, R. M., Steinberg, J. T., and McComas, D. J.: Dispersionless modulations in low-energy solar electron bursts and discontinuous changes in the solar wind electron strahl, J. Geophys. Res.-Space, 109, A05102, doi:10.1029/2003JA010338, 2004a.

Gosling, J. T., Skoug, R. M., McComas, D. J., and Mazur, J. E.: Correlated Dispersionless Structure in Suprathermal Electrons and Solar Energetic Ions in the Solar Wind, Astrophys. J., 614, 412419, doi:10.1086/423368, 2004b.

Greco, A., Servidio, S., Matthaeus, W. H., and Dmitruk, P.: Intermittent structures and magnetic discontinuities on small scales in MHD simulations and solar wind, Planet. Space Sci., 58, 18951899, doi:10.1016/j.pss.2010.08.019, 2010.

Khabarova, O. and Zastenker, G.: Sharp Changes of Solar Wind Ion Flux and Density Within and Outside Current Sheets, Solar Phys., 270, 311-329, doi:10.1007/s11207-011-9719-4, 2011.

Lazarian, A., Vlahos, L., Kowal, G., Yan, H., Beresnyak, A., and de Gouveia Dal Pino, E. M.: Turbulence, Magnetic Reconnection in Turbulent Fluids and Energetic Particle Acceleration, Space Sci. Rev., 173, 557-622, doi:10.1007/s11214-012-9936-7, 2012.

Makov, G. and Payne, M. C.: Periodic boundary conditions in ab initio calculations, Phys. Rev., 51, 4014-4022, doi:10.1103/PhysRevB.51.4014, 1995.

McComas, D. J., Gosling, J. T., Hammond, C. M., Moldwin, M. B., Phillips, J. L., and Forsyth, R. J.: Magnetic reconnection ahead of a coronal mass ejection, Geophys. Res. Lett., 21, 1751-1754, doi:10.1029/94GL01077, 1994.

McComas, D. J., Gosling, J. T., Hammond, C. M., Moldwin, M. B., Phillips, J. L., and Forsyth, R. J.: Reconnection on Open Field Lines Ahead of Coronal Mass Ejections, Space Sci. Rev., 72, 129-132, doi:10.1007/BF00768768, 1995.

Neergaard Parker, L. and Zank, G. P.: Particle Energization during Solar Maximum: Diffusive Shock Acceleration at Multiple Shocks, Astrophys. J., 790, 153, doi:10.1088/0004637X/790/2/153, 2014.

Neergaard Parker, L., Zank, G. P., and Hu, Q.: Particle Acceleration at Quasi-perpendicular Shock Waves: Theory and Observations at $1 \mathrm{AU}$, Astrophys. J., 782, 52, doi:10.1088/0004637X/782/1/52, 2014.

Phan, T. D., Gosling, J. T., Paschmann, G., Pasma, C., Drake, J. F., Øieroset, M., Larson, D., Lin, R. P., and Davis, M. S.: The Dependence of Magnetic Reconnection on Plasma $\beta$ and Magnetic Shear: Evidence from Solar Wind Observations, Astrophys. J. Lett., 719, L199-L203, doi:10.1088/2041-8205/719/2/L199, 2010.

Phan, T. D., Love, T. E., Gosling, J. T., Paschmann, G., Eastwood, J. P., Oieroset, M., Angelopoulos, V., McFadden, J. P., Larson, D., and Auster, U.: Triggering of magnetic reconnection in a magnetosheath current sheet due to compression against the magnetopause, Geophys. Res. Lett., 38, L17101, doi:10.1029/2011GL048586, 2011.

Priest, E. and Forbes, T.: Magnetic Reconnection, Magnetic Reconnection, Cambridge University Press, Cambridge, UK, 612 pp., 2000.

Reames, D. V.: Particle acceleration at the Sun and in the heliosphere, Space Sci. Rev., 90, 413-491, doi:10.1023/A:1005105831781, 1999.
Reames, D. V.: Solar Release Times of Energetic Particles in Ground-Level Events, Astrophys. J., 693, 812-821, doi:10.1088/0004-637X/693/1/812, 2009.

Siversky, T. V. and Zharkova, V. V.: Stationary and impulsive injection of electron beams in converging magnetic field, Astonom. Astrophys., 504, 1057-1070, doi:10.1051/00046361/200912341, 2009a.

Siversky, T. V. and Zharkova, V. V.: Particle acceleration in a reconnecting current sheet: PIC simulation, J. Plasma Phys., 75, 619-636, doi:10.1017/S0022377809008009, 2009b.

Swisdak, M., Opher, M., Drake, J. F., and Alouani Bibi, F.: The Vector Direction of the Interstellar Magnetic Field Outside the Heliosphere, Astrophys. J., 710, 1769-1775, doi:10.1088/0004637X/710/2/1769, 2010.

Verboncoeur, J. P. Langdon, A. B. and Gladd, N. T.: An ObjectOriented Electromagnetic PIC Code, Comp. Phys. Comm., 87, 199-211, doi:10.1016/0010-4655(94)00173-Y, 1995.

Vocks, C. and Mann, G.: Generation of Suprathermal Electrons by Resonant Wave-Particle Interaction in the Solar Corona and Wind, Astrophys. J., 593, 1134-1145, doi:10.1086/376682, 2003.

$\mathrm{Xu}, \mathrm{X}$., Wei, F., and Feng, X.: Observations of reconnection exhausts associated with large-scale current sheets within a complex ICME at 1 AU, J. Geophys. Res.-Space, 116, A05105, doi:10.1029/2010JA016159, 2011.

Zank, G. P. (Ed.): Transport Processes in Space Physics and Astrophysics, Lecture Notes in Physics, Springer Verlag, Berlin, Germany, Vol. 877, doi:10.1007/978-1-4614-8480-6, 2014.

Zank, G. P., Le Roux, J. A., Webb, G. M., Dosch, A., and Khabarova, O.: Particle Acceleration via Reconnection Processes in the Supersonic Solar Wind, Astrophys. J., 797, 28, doi:10.1088/0004-637X/797/1/28, 2014.

Zeiler, A., Biskamp, D., Drake, J. F., Rogers, B. N., Shay, M. A., and Scholer, M.: Three-dimensional particle simulations of collisionless magnetic reconnection, J. Geophys. Res.-Space, 107, 1230, doi:10.1029/2001JA000287, 2002.

Zharkova, V. V. and Agapitov, O. V.: The effect of magnetic topology on particle acceleration in a three-dimensional reconnecting current sheet: a test-particle approach, J. Plasma Phys., 75, 159181, doi:10.1017/S002237780800771X, 2009.

Zharkova, V. V. and Gordovskyy, M.: Particle Acceleration Asymmetry in a Reconnecting Nonneutral Current Sheet, Astrophys. J., 604, 884-891, doi:10.1086/381966, 2004.

Zharkova, V. V. and Gordovskyy, M.: Energy spectra of particles accelerated in a reconnecting current sheet with the guiding magnetic field, Mon. Not. R. Astron. Soc., 356, 1107-1116, doi:10.1111/j.1365-2966.2004.08532.x, 2005.

Zharkova, V. V. and Khabarova, O. V.: Particle Dynamics in the Reconnecting Heliospheric Current Sheet: Solar Wind Data versus Three-dimensional Particle-in-cell Simulations, Astrophys. J., 752, 35, doi:10.1088/0004-637X/752/1/35, 2012.

Zharkova, V. V., Arzner, K., Benz, A. O., Browning, P., Dauphin, C., Emslie, A. G., Fletcher, L., Kontar, E. P., Mann, G., Onofri, M., Petrosian, V., Turkmani, R., Vilmer, N., and Vlahos, L.: Recent Advances in Understanding Particle Acceleration Processes in Solar Flares, Space Sci. Rev., 159, 357-420, doi:10.1007/s11214-011-9803-y, 2011. 